\title{
ОПРЕДЕЛЕНИЕ РАЦИОНАЛЬНЫХ РЕЖИМОВ МЕХАНИЧЕСКОЙ ОБРАБОТКИ ТИТАНОВЫХ КОРПУСНЫХ ЭЛЕМЕНТОВ НЕФТЕГАЗОВОГО ОБОРУДОВАНИЯ КОНЦЕВЫМИ (ПО ГОСТ 23248-78) И ВОЛНОВЫМИ ФРЕЗАМИ
}

\author{
Коровин Георгий Иванович, \\ korovin9@yandex.ru
}

\author{
Гаврилин Алексей Николаевич², \\ gawral@tpu.ru
}

\author{
Петрушин Сергей Игоревич 3 , \\ victory_28@mail.ru
}

\section{Однокопылов Георгий Иванович2, ogiz@yandex.ru}

\author{
Кладиев Сергей Николаевич², \\ kladiev@tpu.ru \\ 1000 ПК «МИОН», \\ Россия, 634034, г. Томск, ул. Вершинина, 46/5, а/я 427. \\ 2 Национальный исследовательский Томский политехнический университет, \\ Россия, 634050, г. Томск, пр. Ленина, 30. \\ 3 Кузбасский государственный технический университет \\ Россия, 652000, г. Кемерово, ул. Весенняя, 28.
}

\begin{abstract}
Актуальность исследования обусловлена тем, что одним из важнейших фракторов безотказной работы нефртегазового оборудования являются требования к коррозионной стойкости. Свойства титановых сплавов: высокая коррозионная стойкость в агрессивных средах, высокая прочность в сочетании с малой плотностью, позволяют получать изделия с большой прочностью и малой массой при работе в условиях агрессивных сред. Однако применение титановых сплавов при изготовлении деталей, работающих в условиях агрессивной среды, в том числе для несртегазового оборудования, ограниченно вследствие неудовлетворительной обрабатываемости резанием, что обусловлено малой теплопроводностью таких сплавов, а также склонностью к интенсивным вибрациям.
\end{abstract}

Объект: производительность и экономическая эфффективность от применения фррез при изготовлении детали типа «корпус» из титанового сплава.

Цель: разработка рациональных режимов механообработки и геометрии инструмента для фрезерования корпусных элементов из титановых сплавов. Рациональные режимы и геометрия фррезы должны обеспечивать максимальную стойкость инструмента, качество механообработки, производительность и экономическую эфффективность.

Методы: проведение производственных испытаний фрез с разной геометрией их режущих кромок методом многофакторного эксперимента с использованием вибродиагностического комплекса для определения зон с минимальным уровнем вибрации при механообработке детали типа «корпус» из титанового сплава.

Результаты. Разработаны рекомендации по снижению вибрации при фррезеровании корпусных элементов из титановых сплавов. Методика позволяет в условиях производства, в стадии отладки технологического процесса, определить рациональные режимы резания по критериям наибольшей производительности и максимальной стойкости инструмента.

\section{Ключевые слова:}

Нефртегазовое оборудование, титановые сплавы, фррезерование, фррезы концевые, производительность, качество механообработки, уровень вибрации, вибродиагностический комплекс, время стойкости инструмента.

\section{Введение}

Высокая коррозионная стойкость титановых сплавов в кислотах позволяет использовать их в нефтегазовом и химическом машиностроении при механообработке: форсунок, работающих в морской воде, сеток фильтров, теплообменников, работающих в азотной кислоте, крыльчатки для центробежных насосов, паровых диффузоров и т. д. Все вышеперечисленные детали изготовлены из нержавеющей стали и при сопоставимой стоимости имеют срок службы в 4...5 раз меныше, чем детали из титановых сплавов.
Положительные качества титановых сплавов, такие как: высокая коррозионная стойкость в агрессивных средах, высокая прочность в сочетании с малой плотностью, позволяют получать изделия с большой удельной прочностью и малой массой.

Применение титановых сплавов при изготовлении деталей, работающих в условиях агрессивной среды, в том числе для нефтегазового оборудования [1], ограниченно вследствие неудовлетворительной обрабатываемости резанием, что обусловлено малой теплопроводностью таких сплавов, а также склонностью к интенсивным вибрациям, что объясняется значи- 
тельными величинами радиальной составляющей силы резания. Следует также отметить, что неустойчивое наростообразование, схватывание выступают дополнительными источниками возбуждения колебаний Снижение уровня вибраций в элементах системы Деталь-Инструмент-Приспособление-Станок (ДИПС) достигается применением комплекса технологическиконструкционных способов виброзащиты, а именно: рациональным подбором режимов механообработки и геометрии инструмента, а также следующими техническими решениями [2-27]:

1) увеличение виброустойчивости и повышение динамических характеристик станков и их узлов;

2) создание виброгасителей для конкретных технологических операций (фрикционных, динамических, гидравлических и т. д.);

3) создание новых методов управления автоколебаниями для увеличения производительности механической обработки, повышения стойкости режущего инструмента и качества обрабатываемой поверхности;

4) проектирование и изготовление режущего инструмента, устойчивого к вибрациям, определение безвибрационных зон режимов резания и т. д.

Зависимость стойкости инструмента или свойства его живучести от интенсивности колебаний ДИПС изучали зарубежные [2-20] и отечественные исследователи [21, 22, 28 29].

Подобные зависимости качественных показателей рабочего процесса от нежелательных колебаний возникают и в сложных электромеханических системах с программным управлением или при передаче энергии переменного тока по кабельной линии для удаленного технологического оборудования, рассматриваемых, например, в публикациях [23-27].

В большинстве исследований получали криволинейную зависимость стойкости от амплитуды колебаний, где с ростом амплитуды стойкость падает.

У некоторых исследователей эта зависимость имеет экстремальный характер, максимальную стойкость получали при амплитуде колебаний $10 . .50$ мкм для быстрорежущего инструмента. Так, увеличение амплитуды автоколебаний в механической системе более некоторых предельных значений приводит к резкому снижению стойкости фрезерного инструмента [2-22, 29].

\section{Методика и результаты исследования}

В данной работе показан многолетний опыт инструментального завода Промышленная Компания (ПК) «МИОН», г. Томск в определении рациональных режимов механообработки на оборудовании заказчика при внедрении производимого компанией инструмента на машиностроительные предприятия. В большинстве случаев при проектировании и изготовлении режущего инструмента под конкретного заказчика конструктору известна только модель станка, в редких случаях - конструкция приспособления, детали, их масса и способы закрепления на станке. Поэтому для проведения испытаний на предприятиях заказчика инструмента для обработки конкретного материала изготавливаются $3 . .5$ фрез с разными гео- метрическими параметрами: окружной шаг, шаг спирали, шаг волны для волновых фрез и т. д.

Далее испытания на оборудовании заказчика проводятся по двум направлениям:

1. В существующий технологический процесс изготовления детали устанавливаются фрезы ПК «МИОН», датчики-акселерометры. Измерения осуществляются с помощью вибродиагностического комплекса «Виброрегистратор-Ф» [30, 31]. При изменении режимов механообработки определяются зоны с минимальным или допускаемым уровнем вибрации. В таблицу параметров заносятся геометрические параметры фрезы. В автоматическом режиме рассчитываются следующие параметры: среднее сечение стружки, число одновременно работающих зубьев, угол встречи с заготовкой, место контакта зуба с заготовкой (точка, линия, пятно контакта), объём снимаемого материала, а также СКЗ амплитуды (виброускорение, виброскорость, виброперемещение).

2. Обработка тестовой партии деталей при серийном производстве. В этом случае имеется возможность влиять на стратегию обработки, изменяя при этом диаметр инструмента, ширину и глубину фрезерования, а также скорость и подачу. В этом случае строится номограмма: ширина фрезы - глубина фрезерования - скорость резания - виброперемещение, с учётом соблюдения критерия допустимого износа инструмента.

На рис. 1 приведена схема механообработки заготовки из титанового сплава испытываемыми фрезами.

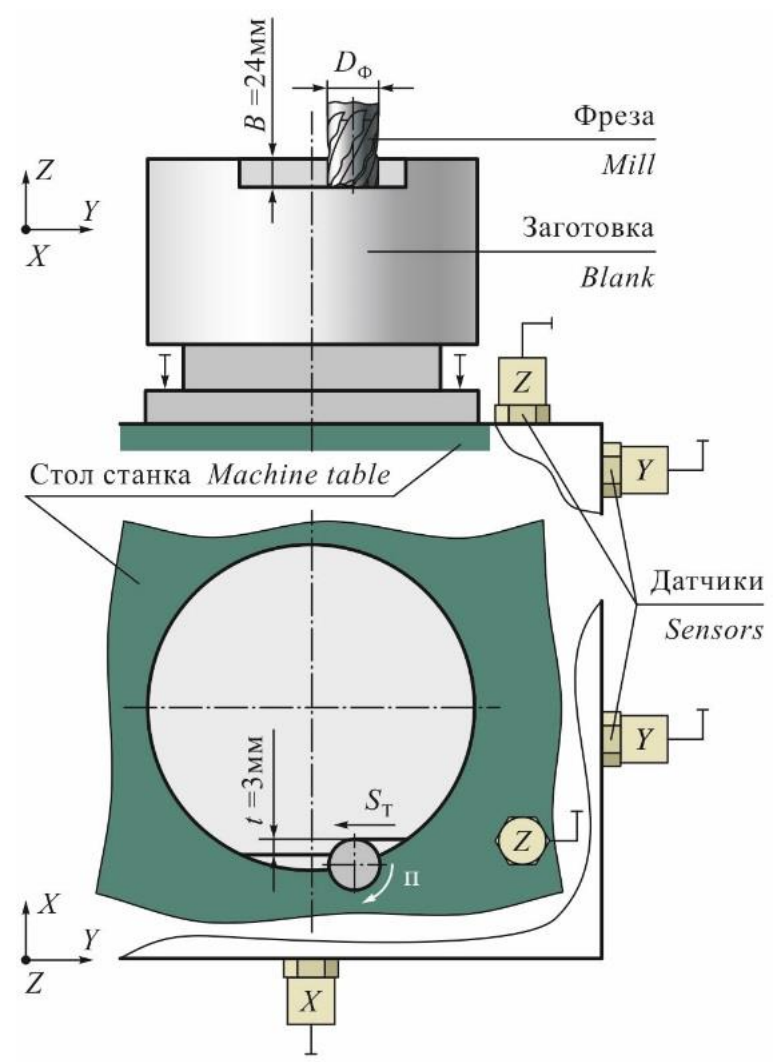

Pис. 1. Схема механообработки заготовки из титанового сплава

Fig. 1. Scheme of machining a titanium alloy blank 
На рис. 2 приведено расположение датчиков на заготовке при фрезеровании на станке MCV-400.

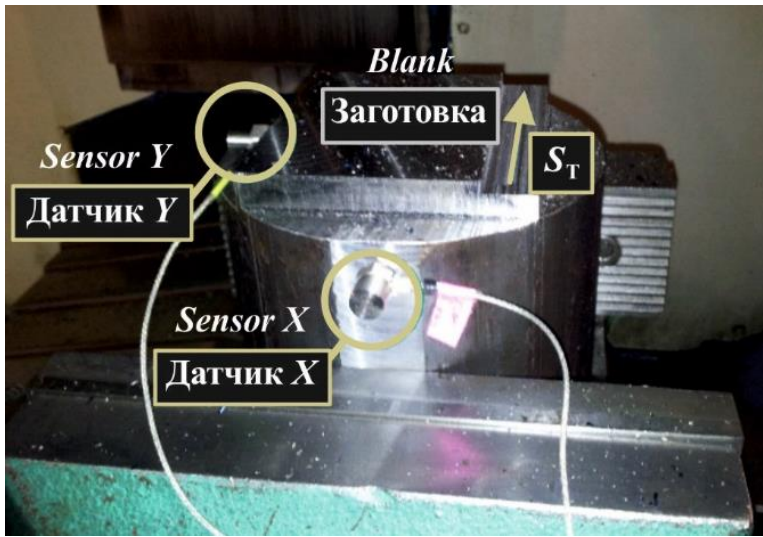

Рис. 2. Расположение датчиков на заготовке при фрезеровании на станке $M C V-400$

Fig. 2. Sensors location on the blank during milling on the MCV-400 machine

На рис. 3 приведены фото: а) волновой фрезы $\varnothing 20$ мм (слева) б) фрезы ТК572 ГОСТ 23248-78 ØØ20 мм, в) конструкция волновой фрезы [30].

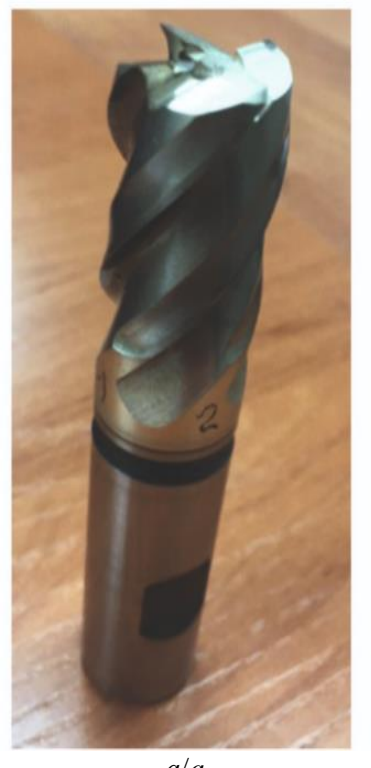

$a / a$

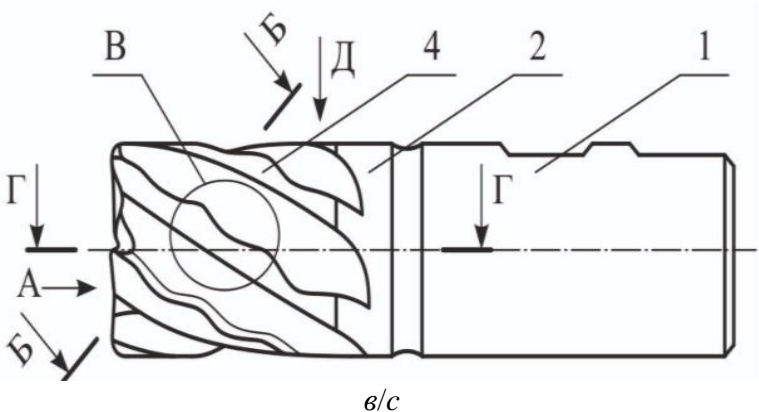

Pис. 3. а) волновая фреза Ø20 мм; б) фреза ТК572 ГОСТ 23248-78 Ø20 мм; в) конструкция волновой фрезы

Fig. 3. a) "Wave type» mill $\varnothing 20 \mathrm{~mm}$; b) TK572 mill according to SS 23248-78 Ø20 $\mathrm{mm}$; c) «Wave type» mill design
Определение рациональных режимов резания для фррез концевых из быстрорежущей стали Р6М5К5 $\varnothing 20$ мм ГОСТ 23248-78 и волновых фрез $\varnothing 20$ мм для изготовления детали «корпус»

Задачи исследования

1. Определить величину виброактивности технологической системы ДИПС, выполнив фрезерование двумя фрезами: по ГОСТ 23248-78 Ø20 мм и волновой фрезой $\varnothing 20$ мм с разной шириной, глубиной фрезерования и скоростью резания при подаче на зуб 0,1 мм.

2. На основании данных вибродиагностического комплекса построить номограммы: ширина фрезерования - $B$ (мм), глубина фрезерования - $t$ (мм), скорость резания - $V$ (м/мин),

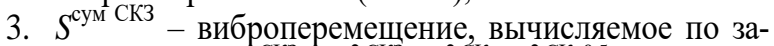

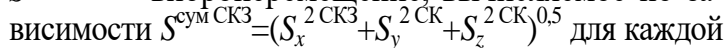
из фрез.

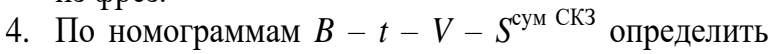
зону режимов обработки (10...50 мкм) с минимальным или допускаемыми параметрами вибрации $[21,22,29]$.

5. По выбранным режимам резания провести механическую обработку деталей «корпус».

6. Рассчитать экономический эффект от использования волновых фрез $\varnothing 20$ мм и фрез по ГОСТ 23248-78 при изготовлении детали «корпус».

7. Данная последовательность действий применяется при внедрении инструмента и его испытании на промышленных предприятиях РФ.

Применяемое оборудование и инструменты

Для определения рациональных режимов резания для изготовления детали «корпус» из титанового сплава ОТ 4 используется следующее оборудование и инструменты:

- станок: фрезерно-расточной четырёхкоординатный, обрабатывающий центр модели MCV-400. Мощность главного привода 12 кВт, вес - $11 \mathrm{~T}$, выпуск 2014 г. Станок соответствует нормам точности, жесткости, осуществлена выборка люфтов в соответствии с паспортными данными станка;

- приспособление: тиски станочные, длина губок $160 \mathrm{~mm}$

- материал: круг 180×200 мм (поковка) из титанового сплава ОТ 4;

- режущий инструмент: фрезы производства завода ПК «МИОН», г. Томск: фреза ГОСТ 23248-78 $\varnothing 20 \times 30 \times 130 \mathrm{KM} 3 Z=4 \gamma_{\mathrm{H}}=15^{\circ} \quad \alpha_{\mathrm{H}}=12^{\circ} \omega=30^{\circ}$; фреза волновая $\varnothing 20 \times 30 \times 100 Z=4 \gamma_{\mathrm{H}}=15^{\circ} \quad \alpha_{\mathrm{H}}=12^{\circ}[28,29]$. Фрезы, используемые в работе, изготавливались методом «вышлифовки и заточки по целому» за одну установку на заточном обрабатывающем центре Walter Power (Германия). Радиальное и осевое биение фрез 2 мкм;

- оправки W20 и КМ3 фирмы «Бизон» (Польша);

- вибродиагностический комплекс «Виброрегистратор-Ф» [31]. Данный комплекс предназначен для регистрации сигналов, поступающих с акселерометров измерительного комплекса в процессе 
проведения испытания фрез, отображения сигналов на мониторе компьютера в виде графиков данных виброускорения, виброперемещения, виброскорости. Частотный диапазон измеряемой вибрации - 5...5000 Гц, частота оцифровки данных - 25 кГц, число используемых каналов - 4;

- датчики-акселерометры модели АР2037 «Глобал Тест».

Схема установки детали, схема фрезерования и места установки акселерометров показаны на рис. 1.

\section{Ход работы и полученные результаты}

Предварительно режимы резания выбирались из практических соображений и данных в работах [2-22, 29] для обработки титановых сплавов ОТ4 быстрорежущими фрезами. Эксперименты проводились по соответствующему плану со скоростями резания: $V=20$, 30, 35, 40 м/мин (Фрезерование попутное, биение фрез на максимальном вылете 2 мкм).

При каждой ширине фрезерования: $B=12,14,20$, 24 мм изменялась глубина фрезерования $t=2 ; 4 ; 5$; 5,$75 ; 6,5 ; 8 ; 8,5 ; 9 ; 9,5 ; 10$ мм. В память виброкомплекса заносились: геометрические параметры фрезы, вылет фрезы, режимы резания, марка обрабатываемого материала и инструмента. Программное обеспечение комплекса рассчитывает: среднее сечение стружки, число эффективных зубьев, угол встречи с заготовкой, точку или пятно контакта торцевого участка зуба с заготовкой.

Таблица 1. Данные виброперемещений в зависимости от режимов резания

Table 1. Vibration displacement data depending on cutting conditions

\begin{tabular}{|c|c|c|c|c|}
\hline \multirow[t]{2}{*}{$\begin{array}{l}B, \mathrm{MM} \\
(\mathrm{mm})\end{array}$} & \multirow[t]{2}{*}{$\begin{array}{l}t, \mathrm{MM} \\
(\mathrm{mm})\end{array}$} & \multirow[t]{2}{*}{$\begin{array}{l}V, \text { м/мин } \\
(\mathrm{m} / \mathrm{min})\end{array}$} & $\begin{array}{c}\text { Фреза волновая } \\
\varnothing 20 \mathrm{mм} \\
\text { Wave mill } \\
\varnothing 20 \mathrm{~mm}\end{array}$ & $\begin{array}{c}\text { Фреза по ГОСТ } \\
23248-78 \varnothing 20 \text { мм } \\
\text { State Standard } \\
\text { mill } \varnothing 20 \mathrm{~mm}\end{array}$ \\
\hline & & & $\begin{array}{r}\text { Вибропє } \\
\text { Vibration displacen }\end{array}$ & $\begin{array}{l}\text { мещение } \\
\text { nt } S^{\text {сум }} \text {, мкм (micron) }\end{array}$ \\
\hline \multirow{7}{*}{12} & 2 & \multirow{7}{*}{40} & 29 & 40 \\
\hline & 4 & & 15 & 22 \\
\hline & 5 & & 29 & 35 \\
\hline & 5,75 & & 32 & 25 \\
\hline & 6,5 & & 35 & 22 \\
\hline & 8 & & 39 & 26 \\
\hline & 10 & & 25 & 25 \\
\hline \multirow{14}{*}{24} & 2 & \multirow{7}{*}{20} & 23 & 1536 \\
\hline & 4 & & 12 & 1030 \\
\hline & 5 & & 15 & 320 \\
\hline & 5,75 & & 14 & 257 \\
\hline & 6,5 & & 20 & 253 \\
\hline & 8 & & 13 & 656 \\
\hline & 10 & & 49 & 223 \\
\hline & 2 & \multirow{7}{*}{40} & 26 & 39 \\
\hline & 4 & & 31 & 25 \\
\hline & 5 & & 47 & 28 \\
\hline & 5,75 & & 29 & 22 \\
\hline & 6,5 & & 39 & 48 \\
\hline & 8 & & 30 & 28 \\
\hline & 10 & & 15 & 120 \\
\hline 14 & 9,5 & 30 & 14 & 912 \\
\hline 20 & 9 & 35 & 20 & 420 \\
\hline \multirow{2}{*}{24} & 9,5 & 40 & 10 & 75 \\
\hline & 8,5 & 20 & 11 & 200 \\
\hline
\end{tabular}

По данным виброактивности программным обеспечением комплекса рассчитываются среднеквадратичные значения (СКЗ виброперемещения, виброскорости и виброускорения) на исследуемом диапазоне частот. В проведенных экспериментах виброактивность проявлялась на зубцовых частотах в полосе 20...45 Гц. В табл. 1 приведены данные виброперемещений $S^{\text {сум СК3 }}$, в зависимости от режимов резания, при которых соответственно изменяются качество механообработки и время стойкости фрезы.

По данным табл. 1 строим номограммы виброактивности для каждой из фрез: фрезы $\varnothing 20$ мм по ГОСТ 23248-78 и волновой фрезы $\varnothing 20$ мм.

На номограммах рис. 4, $a, \sigma$ наблюдается «облако» точек пересечения прямых. Для наглядности точки соединены линиями. Данное «облако» ограничиваем четырехугольником $E M H G$, левая сторона которого максимальная граница вибрации, правая - минимальная граница вибрации, нижняя сторона - минимальная ширина и глубина фрезерования, верхняя - максимальная ширина и глубина фрезерования. Таким образом, в четырехугольнике $E M H G$ находятся рекомендуемые режимы резания с соответствующими допускаемыми уровнями вибрации [21, 22]. Данный диапазон впоследствии можно расширить или уменьшить в зависимости от стойкостных испытаний.

\section{Алгоритм построения номограмм}

Алгоритм нанесения точек на номограмму, по данным из табл. 1:

1. На оси «B, мм» (ширина фрезерования) отмечаем необходимую точку $A=24$ мм.

2. На оси «t, мм» (глубина фрезерования) отмечаем необходимую точку $C=9,5 \mathrm{mм}$.

3. Соединяем в номограмме точки $A$ и $C$. Получаем отрезок $A C$.

4. На оси «V, м/мин» (скорость резания) отмечаем необходимую точку $D=40 \mathrm{~m} / \mathrm{\text {ин. }}$

5. На оси « $\log _{2} S^{\text {сум СК3 }}$, мкм» (виброперемещение) отмечаем необходимую точку $E=5$ мм.

6. Соединяем в номограмме точки $D$ и $E$. Получаем отрезок $D E$.

7. Пересечение отрезков $A C$ и $D E$ дает точку $F$.

8. Полученная точка $F$ входит в область $E N H G$.

9. Внутри области $E N H G$ находится виброустойчивая зона режимов резания.

10. Область $E N H G$ на номограмме (рис. 4) построена по следующему алгоритму:

10.1. На оси «В» (ширина фрезерования), в соответствии с принятой шириной фрезерования (диапазон значений $B=12,14,20,24$ мм), отмечаем точку $L=12$ мм - минимальное, и $A=24$ мм максимальное значение ширины фрезерования.

10.2. На оси «t, мм» (глубина фрезерования) в соответствии с принятой глубиной фрезерования (диапазон значений $t=2 ; 4 ; 5 ; 5,75 ; 6,5 ; 8$; 8,$5 ; 9 ; 9,5 ; 10$ мм) отмечаем $K=2$ мм - минимальное, и $P=10$ мм - максимальное значение глубины фрезерования.

10.3. Соединяем точки $L$ и $K$. Получаем отрезок $L K$. 
10.4. Соединяем точки $A$ и $P$. Получаем отрезок $A P$.

10.5. На оси «V» (скорость резания), в соответствии с принятой скоростью резания (диапазон значений $V=20,30,35,40 \mathrm{~m} /$ мин), откладываем $X=20 \mathrm{м} /$ мин - минимальное, и $J=40$ м/мин максимальное значение скорости резания.

10.6. На оси « $\log _{2} S^{\text {сум СК3 }}$, мкм» (виброперемещение), в соответствии с зоной рациональных по критерию стойкости амплитуд автоколебаний для быстрорежущего инструмента (фрезы концевые), откладываем $Z=2$ мкм - минимальное,

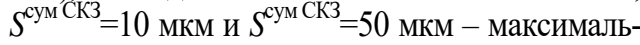
ные значения виброперемещения [21].
10.7. Соединяем точки $X$ и $Z$. Получаем отрезок $X Z$. 10.8. Соединяем точки $J$ и $S$. Получаем отрезок $J S$.

10.9. Пересечение отрезков $A P$ и $J S$ дает точку $E$. Пересечение отрезков $A P$ и $X Z$ дает точку $M$. Пересечение отрезков $L K$ и $J S$ дает точку $G$. Пересечение отрезков $L K$ и $X Z$ дает точку $H$.

10.10.Соединяем точки $E$ и $M$, получаем отрезок $E M$. 10.11.Соединяем точки $M$ и $H$, получаем отрезок $M H$. 10.12.Соединяем точки $H$ и $G$, получаем отрезок $H G$. 10.13 .Соединяем точки $G$ и $E$, получаем отрезок $G E$. 10.14.В результате соединения отрезков получаем область рациональных значений режимов резания $E M H G$.

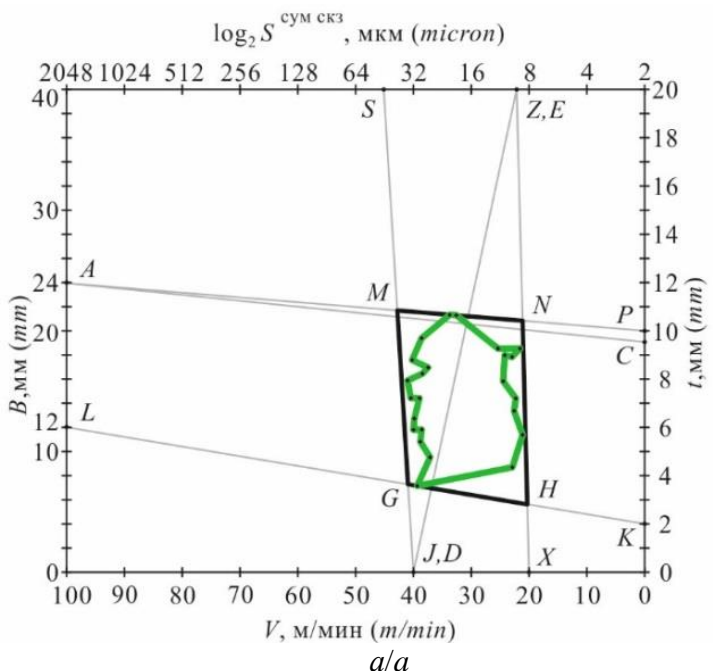

ala

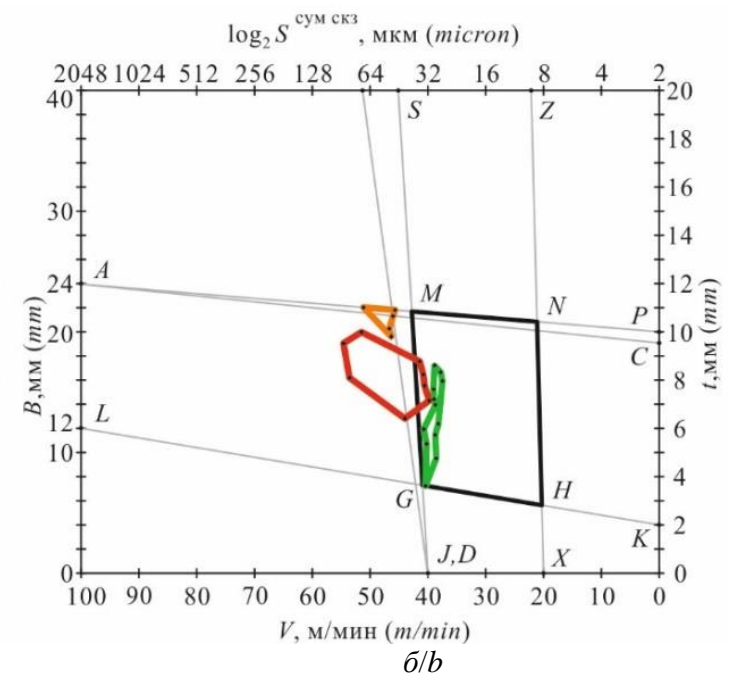

$\sigma / b$

- Оптимальный уровень вибрации (от 10 до 50 мкм)/Optimal vibration level (from 10 to 50 microns).

- Допустимый уровень вибрации (от 50 до 250 мкм)/Allowable vibration level (from 50 to 250 microns).

- Предельный уровень вибрации (от 250 до 1000 мкм)/Limit vibration level (from 250 to 1000 microns).

Puc. 4. Номограммы: а) для волновой фрезы; б) для фрезы по ГОСТ ТК 572

Fig. 4. Nomograms: a) for a wave type mill; b) for a mill according to SS TK 572

Из анализа номограмм рис. 4, $a, 6$ можно сделать вывод о том, что применение волновой фрезы при черновой механообработке и максимальной производительности с заданными ограничениями уровней

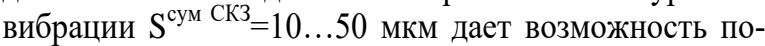
вышения стойкости инструмента $T_{\text {ст }}$ (или $L_{\text {ст }}$ ) (табл. 2 ) более чем в 3 раза для получения необходимого качества поверхности обработанной детали из титана в пределах $R_{z}=10 \ldots 60$ мкм по сравнению со стандартной фрезой. Следует отметить, что при указанных режимах и производительности механообработки качество обработанной детали стандартной фрезой составляло $R_{z}=50 \ldots 180$ мкм. Полученные номограммы (рис. 4) остаются у технологов предприятия-заказчика инструмента и могут впоследствии дополняться виброустойчивыми зонами при осевом врезании, врезании под углом, при обходах радиусов и т. д.

\section{Рекомендации по снижению вибрации системы ДИПС}

1. Жесткость системы ДИПС должна быть не менее $20 \ldots 100 \mathrm{H} /$ мкм.

2. Требуется контроль параметров: скорость резания $V$ (м/мин), ширина фрезерования $B$ (мм), глубина

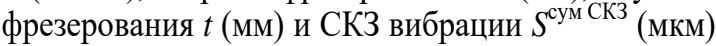

При возрастании вибрации $S^{\text {сум СК3 }}$ выше допускаемых $10 \ldots 50$ мкм изменять указанные параметры в соответствии с табл. 1 или номограммой рис. 4.

Следует отметить, что полученные номограммы будут являться действительными только для конкретной технологической системы, включая вылет инструмента и качество обрабатываемого материала, т. е. экономически выгодны для серийного, крупносерийного и массового производства.

Для дальнейшей работы выбирались режимы механообработки по критерию наибольшей производительности и фрезы, имеющие минимальную виброактивность. Данными фрезами выполнялись работы по удалению основного припуска для придания необходимой формы детали «корпус». На этом этапе будет определяться стойкость фрез.

Для дальнейшей работы были выбраны два типа фрез с режимами обработки, где наблюдалась минимальная виброактивность: волновая фреза $\varnothing 20$ мм и фреза Ø20 мм по ГОСТ 23248-78.

В дальнейшем данные фрезы с соответствующими режимами резания (табл. 1) использовались в технологическом процессе изготовления детали «корпус». Заготовкой являлась поковка $\varnothing 180 \times 25$ из титанового 
сплава ОТ4 производства Верхне-Салдинского Металлургического Комбината (ВСМПО). Предельным износом являлся износ по задней грани, равный

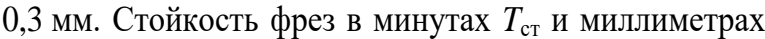
$L_{\text {ст }}$ показана в табл. 2.

Таблица 2. Сравнительная эффективность двух фрез Table 2. Comparative efficiency of two mills

\begin{tabular}{|c|c|c|c|}
\hline \multicolumn{2}{|c|}{$\begin{array}{c}\text { Обозначение фрезы } \\
\text { Mill type }\end{array}$} & $\begin{array}{c}\text { Волновая } \\
\text { фреза } \\
\text { Wave mill } \\
\varnothing 20 \text { мм (mm) }\end{array}$ & $\begin{array}{c}\text { Фреза } \varnothing 20 \text { мм } \\
\text { ГОСТ 23248-78 } \\
\text { State Standard } \\
\text { mill }\end{array}$ \\
\hline \multirow{4}{*}{$\begin{array}{l}\text { Режимы } \\
\text { обработки } \\
\text { Machining } \\
\text { modes }\end{array}$} & 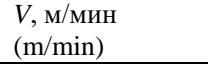 & \multicolumn{2}{|c|}{$\begin{array}{lllll} & \\
\end{array}$} \\
\hline & $B$, мм $(\mathrm{mm})$ & \multicolumn{2}{|r|}{24} \\
\hline & $T$, мм $(\mathrm{mm})$ & \multicolumn{2}{|r|}{9,5} \\
\hline & 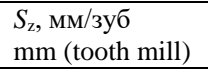 & \multicolumn{2}{|r|}{0,1} \\
\hline \multicolumn{2}{|c|}{$\begin{array}{l}S^{\text {сум ск3 }}, \text { мкм (micron) } \\
\text { Виброперемещение } \\
\text { Vibration displacement }\end{array}$} & 5 & 15 \\
\hline \multicolumn{2}{|c|}{$\begin{array}{l}\Pi_{\text {Д}}, \text { мм (mm) } \\
\text { Путь при изготовлении } \\
\text { одной детали } \\
\text { Distance during a detail } \\
\text { manufacturing }\end{array}$} & 520 & 520 \\
\hline \multicolumn{2}{|c|}{$\begin{array}{l}Q_{д}, \text { шт. (itm.) } \\
\text { Количество деталей за период } \\
\text { стойкости } \\
\text { Parts number for the period of life }\end{array}$} & 35,2 & 11,4 \\
\hline \multicolumn{2}{|c|}{$\begin{array}{l}T_{\text {ст }}, \text { мин }(\min ) \\
\text { Bремя стойкости } \\
\text { Durability time }\end{array}$} & 71,8 & 23,3 \\
\hline \multicolumn{2}{|c|}{$\begin{array}{l}L_{\text {ст }}, \text { мм }(\mathrm{mm}) \\
\text { Стойкость в пройденном } \\
\text { расстоянии } \\
\text { Durability in the distance } \\
\end{array}$} & 18298 & 5932 \\
\hline
\end{tabular}

Экономическая эффективность от применения фрез ГОСТ 23248-78 Ø20 мм и волновых фрез $\varnothing 20$ мм при изготовлении детали «корпус» из титанового сплава ОТ4

Из табл. 1, 2 видно, что режимы обработки практически одинаковы для всех фрез (припуск по контуру 0,5 мм для фрез волновых $\varnothing 20$ мм и ГОСТ 23248 $78 \varnothing 20$ мм оставлен на чистовую операцию), поэтому в расчётах использован путь, пройденный каждой фрезой за время обработки одной детали $-\Pi_{д}=520 \mathrm{Mм}$

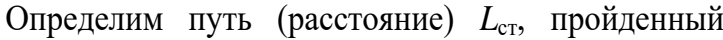
каждой из фрез до износа $h_{3}=0,3$ мм (табл. 2$)$ :

$$
L_{\text {ст }}=S_{\text {мин }} \cdot T_{\text {ст }},
$$

где $S_{\text {мин }}-$ минутная подача, мм/мин; $T_{\text {ст }}-$ стойкость фрезы, мин.

$$
S_{\text {мин }}=S_{z} \cdot Z \cdot n,
$$

где $S_{z}$ - подача на зуб, мм; $Z$ - число зубьев фрезы; $n-$ число оборотов шпинделя, об/мин;

Количество деталей $Q_{\text {д }}$ обработанных каждой фрезой за период стойкости, определено по формуле:

$$
Q_{\text {д }}=L_{\text {ст }} / \Pi_{\text {д }},
$$

где $\Pi_{д}-$ путь, пройденный фрезой при изготовлении одной детали, мм (табл. 2).

Количество фрез $K_{\phi р}$, необходимых для изготовления партии деталей $N=100$ штук.

$$
\begin{gathered}
K_{\phi р}=N / Q_{\text {д }}, \\
K_{\text {волна }}=100 / 35,2=2,84 \text { фрезы; } \\
K_{\text {Гост }}=100 / 11,4=8,77 \text { фрезы. }
\end{gathered}
$$

Стоимость $C_{\phi p}$ фрез, произведённых на предприятии ООО ПК «МИОН»:

$$
C_{\text {волна }}=5590 \text { р.; } C_{\text {ГОСТ }}=2442 \mathrm{p} .
$$

где $C_{\text {волна }}, C_{\text {Гост }}-$ стоимость волновой и стандартной фрез соответственно.

Затраты 3 и на инструмент при черновом фрезеровании детали «корпус» определяются по формуле:

$$
3_{\text {и }}=K_{\phi р} \cdot C,
$$

где $K_{\phi p}-$ количество фрез, необходимых для изготовления партии деталей; $C_{\phi р}$ - цена фрезы (р.).

$$
\begin{gathered}
3_{\text {волна }}=2,84 \times 5990=17011,6 \mathrm{p} . ; \\
3_{\text {ГОСТ }}=8,77 \times 2442=21416,34 \mathrm{p} .
\end{gathered}
$$

Экономическая эффективность Э от использования волновой фрезы $\varnothing 20$ мм с режимами обработки по сравнению с фрезой Ø20 мм ГОСТ 23248-78 с режимами обработки (табл. 1) при изготовлении партии деталей:

$$
\begin{gathered}
Э_{\text {волна }}=3_{\text {ГОСТ }} / 3_{\text {волна }} ; \\
Э_{\text {волна }}=21416,34 / 17011,6=1,259 .
\end{gathered}
$$

По результатам работы и многочисленным ресурсным испытаниям на ВСМПО-АВИСМА г. Верхняя Салда и др. внесена поправка в ГОСТ 23248-78 «Фрезы концевые для обработки деталей из высокопрочных сталей и титановых сплавов на станках с программным управлением»: «...2. Фрезы с длиной режущей части $l=3 D$ рекомендуются для применения с волнообразной режущей кромкой по технической документации, утверждённой в установленном порядке» [30]. Также разработаны технические условия на волновые фрезы ТУ 3918-018-36293294-2015.

Следует отметить, что при механообработке жаропрочных и титановых сплавов в точке соприкосновения инструмента и детали выделяется значительное количество тепла, обусловленное адгезионными процессами, малой теплопроводностью таких сплавов, а также значительными величинами радиальной составляющей силы резания и неустойчивым наростообразованием, схватыванием.

Это отмечено во введении, при этом указанные факторы выступают дополнительными источниками возбуждения автоколебаний.

\section{Выводы}

1. Разработанная методика с использованием вибродиагностического комплекса «ВиброрегистраторФ» для построения номограмм позволяет в условиях производства на стадии отладки технологического процесса определить рациональные режимы резания по критериям наибольшей производительности и максимальной стойкости инструмента. 
2. Экономический эффект от применения предложенного технического решения, посчитанный по формуле (6), непосредственно связан с параметрами виброактивности технологической системы: чем ниже виброактивность, тем выше стойкость инструмента и тем больший эффект от его использования. Снижение виброактивности волновых фрез по сравнению с фрезами по ГОСТ 23248-78, в свою очередь, связано с работой каждого зуба по менее наклёпанному слою.

3. На техническое решение по волновой фрезе получен патент на изобретение № 2601525 РФ, а также по инициативе ООО «Промышленная компания «МИОН» внесена поправка в ГОСТ 23248-78

\section{СПИСОК ЛИТЕРАТУРЬ}

1. Винокурова И.М., Коротов В.В. Использование титановых сплавов в целях повышения эксплуатационных параметров нефтегазового оборудования // Современные тенденции развития науки и технологий. - 2016. - № 11-3. - С. 28-33.

2. Investigation of tool wear and chip morphology in dry trochoidal milling of titanium alloy Ti-6Al-4V / D. Liu, Y. Zhang, M. Luo, D. Zhang // Materials. - 2019. - V. 12. - Iss. 12. - P. 1937-1949 URL: https://doi.org/10.3390/ma12121937 (дата обращения 15.05.2021)

3. Investigation on wear behavior of cryogenically treated Ti-6Al-4V titanium alloy under dry and wet conditions / Y. Li, X. Wang, S. Yang, L. Hou, Y. Wei, Z. Zhang, X. Yang // Materials. 2019. - V. 12. - Iss. 18. - P. 2850-2863. URL: https://doi.org/ $10.3390 / \mathrm{ma1} 2182850$ (дата обращения 15.05.2021).

4. Investigation of cutting force in longitudinal-torsional ultrasonicassisted milling of Ti-6Al-4V / Y. Niu, F. Jiao, B. Zhao, G. Gao // Materials. - 2019. - V. 12. - Iss. 12. - P. 1955-1969. URL: https://doi.org/10.3390/ma12121955

5. Bari P., Law M., Vakhi P. Geometric models of non-standard toothed end mills // International Journal of Advanced Manufacturing Technology. - 2020. - V. 111. - Iss. 11-12. P. 3319-3342.

6. Modelling and simulation of effect of ultrasonic vibrations on machining of Ti6Al4V / Sandip Patil, Shashikant Joshi, Asim Tewari, Suhas S Joshi // Affiliations expand. - 2014. - V. 54. Iss. 2. - P. 694-705

7. Feasibility study of ultrasonic elliptical vibration-assisted reaming of carbon fiber reinforced plastics/titanium alloy stacks / D. Geng, D. Zhang, Z. Li, D. Liu // Ultrasonics. - 2017. - V. 75. - P. 80-90.

8. Eun Jung Kim, Choon Man Lee. A study on the optimal machining parameters of the induction assisted milling with Inconel 718 // Materials. - 2019. - V. 12. - Iss. 2. - P. 233-248. URL: https://doi.org/10.3390/ma12020233 (дата обращения 15.05.2021)

9. Machining of titanium metal matrix composites: progress overview / C. Escaich, Z. Shi, L. Baron, M. Balazinski // Materials. - 2020. V. 13. - Iss. 21. - P. 5011-5026. URL: https://doi.org/ 10.3390/ma13215011 (дата обращения 15.05.2021).

10. Study of the effects of initial cutting conditions and transition period on ultimate tool life when machining Inconel 718 / M. Memarianpour, S.A. Niknam, S. Turenne, M. Balazinski // Materials. - 2021. - V. 14. - Iss. 3. - P. 592-604. URL https://doi.org/10.3390/ma14030592 (дата обращения 15.05.2021)

11. Rao Charitha, Rao Shrikantha, Herbert Mervin. Performance improvement studies for cutting tools with a perforated surface when turning titanium alloy // MATEC Web of Conferences. 2018. - V. 144, 03003. URL: https://doi.org/10.1051/ matecconf/201814403003 (дата обращения 15.05.2021).

12. Experimental characterization of tool wear morphology and cutting force profile in dry and wet turning of titanium metal matrix composites (Ti-MMCs) / M. Safavi, M. Balazinski, H. Mehmanparast, S.A. Niknam // Metals. - 2020 - V. 10. Iss. 11. - P. 1459-1472. URL: https://doi.org/10.3390/met10111459 (дата обращения 15.05.2021).
«Фрезы концевые для обработки деталей из высокопрочных сталей и титановых сплавов на станках с программным управлением».

4. Предложенное техническое решение позволяет повысить эффективность механообработки титановых сплавов корпусных элементов не только для нефтегазового оборудования, но и для других отраслей промышленности: медицинской, авиационной, атомной и т. д.

5. Особенности геометрии волновой фрезы и выбор оптимальных режимов механообработки титановых сплавов с её участием позволяют значительно снизить уровень вибрации в системе «детальинструмент», что является предметом «ноу-хау».

13. Erol Kilickap, Ahmet Yardimeden, Yahya Hışman Çelik. Mathematical modelling and optimization of cutting force, tool wear and surface roughness by using artificial neural network and response surface methodology in milling of Ti-6242S // Applied Sciences. - 2017. - V. 7. - Iss. 10. - P. 1064-1075. URL: https://doi.org/10.3390/app7101064 (дата обращения 15.05.2021)

14. Ramesh Rudrapati, Pradip Kumar Pal, Asish Bandyopadhyay. Vibration in traverse cut cylindrical grinding - experiments and analysis // Advanced Materials Research. - 2011. - V. 264-265. P. 1124-1129.

15. Çelik Y.H., Yildiz H., Özek H.C. Effect of cutting parameters on workpiece and tool properties during drilling of Ti-6Al-4V // Materials Testing. - 2016. - V. 58. - Iss. 6. - P. 519-525.

16. Tehranizadeh F., Koca R., Budak E. Investigating effects of serration geometry on milling forces and chatter stability for their optimal selection // International Journal of Machine Tools and Manufacture. - 2019. - V.144. - art. N. 103425. URL: https://doi.org/10.1016/j.ijmachtools.2019.103425 (дата обращения 15.05.2021).

17. Bari P., Law M., Wahi P. Improved chip thickness model for serrated end milling // CIRP Journal of Manufacturing Science and Technology. - 2019. - V. 25. - P. 36-49. URL: https://doi.org/10.1016/j.cirpj.2019.03.001 (дата обращения 15.05.2021)

18. Guo Y., Lin B., Wang W. Modeling of cutting forces with a serrated end mill // International Journal of Machine Tools and Manufacture. - 2019. - V. 144. - art. N. 103425. URL: https:// doi.org/10.1155/2019/1796926 (дата обращения 15.05.2021).

19. Dombovari Z., Altintas Y., Stepan G. The effect of serration on mechanics and stability of milling cutters // International Journal of Machine Tools and Manufacture. - 2010. - V. 50. - Iss. 6. P. 511-520. URL: https://doi.org/10.1016/j.ijmachtools.2010.03.006 (дата обращения 15.05.2021)

20. Experimental studies of stabilization of boring cutter form building top oscillation / M.R. Sikhimbaev, K.T. Sherov, O.M. Zharkevich, A.K. Sherov, Y.O. Tkachyova // Journal of Vibroengineering. - 2012. - V. 14. - Iss. 2. - P. 661-670.

21. Application of integrated methods to improve the technological properties of steel / S.N. Fedoseev, D.V. Valuev, R.A. Mamadaliev, P.N. Sokolov // Key Engineering Materials. 2017. - V. 736. - P. 110-115. URL: https://doi.org/10.4028/www. scientific.net/KEM.736.110 (дата обращения 15.05.2021).

22. Лукьянов А.В., Алейников Д.П., Вищенко М.В. Исследование пространственных вибраций и сил при высокопроизводительном фрезеровании // Транспортная инфраструктура Сибирского региона. - 2017. - Т. 2. - С. 598-603.

23. Bukreev V.G., Lyapushkin S.V., Edgulov M.M. Accuracy enhancement of automated system for multicomponent batching of bulk materials // MATEC Web of Conferences. - 2015. - V. 37. 01012.

24. Букреев В.Г., Шандарова Е.Б., Рулевский В.М. Многомерная модель системы электропитания погружного технологического оборудования // Известия Томского политехнического университета. Инжиниринг георесурсов. - 2018. - Т. 329. - № 4. C. $119-131$. 
25. The power supply system model of the process submersible device with AC power transmission over the cable-rope / V.M. Rulevskiy, V.G. Bukreev, E.O. Kuleshova, S.M. Shandarov, Yu.Z. Vasilyeva // IOP Conference Series: Materials Science and Engineering. 2017. - V. 177 (1). - 012098.

26. Nizhegorodov A.I., Zvezdin A.V. Transformation of vermiculite energy into mechanical transformation energy during firing in electric furnaces with a "zero» module // Refractories and Industrial Ceramics. - 2016. - V. 57. - Iss. 3. - P. 239-245.

27. Influence of design methods a discrete model of separately excited DC motor on parameters estimation / I. Vajda, A. Glazyrin, I. Ustinova, E. Bolovin // Acta Polytechnica Hungarica. - 2018. V. 15. - Iss. 6. - P. 219-233.

28. Фреза концевая для обработки титановых сплавов: пат. RU 2014127926 А. Рос. Федерация № 2014127926/02; заявл. 07.07.14; опубл. 27.01.2016. - Бюл. № 3. - 1 с.
29. Korovin G.I., Petrushin S.I., Gubaidulina R.H. Machining of titanium alloys with wave milling cutters // Materials Science Forum. - 2018. - 927. - P. 79-85.

30. Фрезы концевые из быстрорежущей стали для обработки титановых сплавов. Официальный сайт ООО ПК «МИОН». URL: http://pkmion.ru/catalog/dlya-obrabotki-titanovykh-splavov/710dlya-obrabotki-titanovykh-splavov/ (дата обращения 15.05.2021)

31. Гаврилин А.Н., Виноградов А.А., Серебряков К.В. Виброрегистратор-Ф. Свидетельство о регистрации программы для ЭВМ Российской Федерации № 2014661189. Дата регистрации 24.10.2014.

Поступила 09.06.2021 г.

\section{Информация об авторах}

Коровин Г.И., технический директор ООО ПК «МИОН».

Гаврилин A.H., кандидат технических наук, доцент отделения материаловедения Инженерной школы новых производственных технологий Национального исследовательского Томского политехнического университета.

Петрушин С.И., доктор технических наук, профессор, Кузбасский государственный технический университет.

Однокопылов Г.И., доктор технических наук, профессор отделения электроэнергетики и электротехники Инженерной школы энергетики Национального исследовательского Томского политехнического университета.

Кладиев $\boldsymbol{C . H . , ~ к а н д и д а т ~ т е х н и ч е с к и х ~ н а у к , ~ д о ц е н т ~ о т д е л е н и я ~ э л е к т р о э н е р г е т и к и ~ и ~ э л е к т р о т е х н и к и ~ И н ж е н е р - ~}$ ной школы энергетики Национального исследовательского Томского политехнического университета. 


\title{
DETERMINING RATIONAL MODES OF MECHANICAL PROCESSING OF TITANIUM BODY ELEMENTS OF OIL AND GAS EQUIPMENT BY END-END MACHINES (ACCORDING TO SS 23248-78) AND WAVE-SHAPED CUTTERS
}

\author{
Georgy I. Korovin 1 , \\ korovin9@yandex.ru
}

\author{
Alexey N. Gavrilin², \\ gawral@tpu.ru
}

\author{
Sergey I. Petrushin ${ }^{3}$, \\ victory_28@mail.ru
}

Georgy I. Odnokopylov², ogiz@yandex.ru

\author{
Sergey N. Kladiev², \\ kladiev@tpu.ru \\ 1 Industrial Company LLC « MION», \\ 46/5, Vershinin street, Tomsk, 634034, Russia. \\ 2 National Research Tomsk Polytechnic University, \\ 30, Lenin avenue, Tomsk, 634050, Russia. \\ 3 Kuzbass State Technical University, \\ 28, Vesennya street, Kemerovo, 650000, Russia.
}

The relevance of the research is caused by the fact that one of the most important factors in the failure-free operation of oil and gas equipment is the corrosion resistance requirements. Titanium alloys properties like high corrosion resistance in corrosive environments and high strength combined with low density make it possible to obtain products with high strength and low weight when operating in corrosive environments. However, titanium alloys usage in the manufacture of details that operate in an aggressive environment, including for oil and gas equipment, is limited due to both low machinability and low thermal conductivity, as well as the technological system predisposition to intense fluctuations.

Object: productivity and economic efficiency from using mills for manufacturing hull details like «case» type from titanium alloy.

The main aim of the research is a development of rational machining modes and tool geometry for milling hull elements from titanium alloys. That can ensure maximum tool life, machining quality, productivity and economic efficiency.

Methods: production tests of mills with different geometry of cutting edges by the multifactor experiment method using a vibro-diagnostic complex for determine the minimum vibration level zones during machining of the «case» type part from titanium alloy.

Results. The authors have developed the recommendations to reducing vibration during milling hull elements from titanium alloys. This method allows finding the rational cutting conditions according to both the highest productivity and maximum tool life criteria in production conditions during the stage of debugging the technological process.

Key words:

Oil and gas equipment, titanium alloys, milling, end mills, productivity, machining quality, vibration level, vibro-diagnostic complex, tool life.

\section{REFERENCES}

1. Vinokurova I.M., Korotov V.V. Ispolzovanie titanovykh splavov v tselyakh povysheniya ekspluatacionnykh parametrov neftegazovogo oborudovaniya [Titanium alloys using in order to improve the operational parameters of oil and gas equipment]. Modern trends in the development of science and technologies, 2016, no. 11-3, pp. 28-33.

2. Liu D., Zhang Y., Luo M., Zhang D. Investigation of tool wear and chip morphology in dry trochoidal milling of titanium alloy Ti-6Al-4V. Materials, 2019, vol. 12, no. 12, pp. 1937-1949. Available at: https://doi.org/10.3390/ma12121937 (accessed 15 May 2021)

3. Li Y., Wang X., Yang S., Hou L., Wei Y., Zhang Z., Yang X. Investigation on wear behavior of cryogenically treated Ti-6Al-4V titanium alloy under dry and wet conditions. Materials, 2019, vol. 12, no. 18, pp. 2850-2863. Available at: https://doi.org/10.3390/ma12182850

4. Niu Y., Jiao F., Zhao B., Gao G. Investigation of cutting force in longitudinal-torsional ultrasonic-assisted milling of Ti-6Al-4V. Materials, 2019, vol. 12, no. 12, pp. 1955-1969. Available at: https://doi.org/10.3390/ma12121955 (accessed 15 May 2021).
5. Bari P., Law M., Vakhi P. Geometric models of non-standard toothed end mills. International Journal of Advanced Manufacturing Technology, 2020, vol. 111, no. 11-12, pp. 3319-3342.

6. Sandip Patil, Shashikant Joshi, Asim Tewari, Suhas S Joshi. Modelling and simulation of effect of ultrasonic vibrations on machining of Ti6A14V. Affiliations expand, 2014, vol. 54, no. 2, pp. 694-705.

7. Geng D., Zhang D., Li Z., Liu D. Feasibility study of ultrasonic elliptical vibration-assisted reaming of carbon fiber reinforced plastics/titanium alloy stacks. Ultrasonics, 2017, vol. 75, pp. 80-90.

8. Eun Jung Kim, Choon Man Lee. A study on the optimal machining parameters of the induction assisted milling with Inconel 718. Materials, 2019, vol. 12, no. 2, pp. 233-248. Available at: https://doi.org/10.3390/ma12020233 (accessed 15 May 2021).

9. Escaich C., Shi Z., Baron L., Balazinski M. Machining of titanium metal matrix composites: progress overview. Materials, 2020, vol. 13, no. 21, pp. 5011-5026. Available at: https://doi.org/ 10.3390/ma13215011 (accessed 15 May 2021).

10. Memarianpour M., Niknam S.A., Turenne S., Balazinski M. Study of the effects of initial cutting conditions and transition period on ultimate tool life when machining Inconel 718. Materials, 2021, 
vol. 14, no. 3, pp. 592-604. Available at: https://doi.org/10.3390/ ma14030592

11. Rao Charitha, Rao Shrikantha, Herbert Mervin. Performance improvement studies for cutting tools with a perforated surface when turning titanium alloy. MATEC Web of Conferences, 2018, vol. 144, 03003. Available at: https://doi.org/10.1051/matecconf/ 201814403003 (accessed 15 May 2021).

12. Safavi M., Balazinski M., Mehmanparast H., Niknam S.A. Experimental characterization of tool wear morphology and cutting force profile in dry and wet turning of titanium metal matrix composites (Ti-MMCs). Metals, 2020, vol. 10, no. 11, pp. 1459-1472. Available at: https://doi.org/10.3390/met10111459

13. Erol Kilickap, Ahmet Yardimeden, Yahya Hısman Celik. Mathematical modelling and optimization of cutting force, tool wear and surface roughness by using artificial neural network and response surface methodology in milling of Ti-6242S. Applied Sciences, vol. 7, no. 10, pp. 1064-1075. Available at: https://doi.org/ 10.3390/app7101064 (accessed 15 May 2021).

14. Ramesh Rudrapati, Pradip Kumar Pal, Asish Bandyopadhyay. Vibration in Traverse Cut Cylindrical Grinding - Experiments and Analysis. Advanced Materials Research, 2011, no. 264-265, pp. 1124-1129.

15. Çelik Y.H., Yildiz H., Özek H.C. Effect of cutting parameters on workpiece and tool properties during drilling of Ti-6Al-4V. Materials Testing, 2016, vol. 58, no. 6, pp. 519-525.

16. Tehranizadeh F., Koca R., Budak E. Investigating effects of serration geometry on milling forces and chatter stability for their optimal selection. International Journal of Machine Tools and Manufacture, 2019, vol. 144, art. no. 103425. Available at: https:// doi.org/10.1016/j.ijmachtools.2019.103425 (accessed 15 May 2021).

17. Bari P., Law M., Wahi P. Improved chip thickness model for serrated end milling. CIRP Journal of Manufacturing Science and Technology, 2019, vol. 25, pp. 36-49. Available at: https:// doi.org/10.1016/j.cirpj.2019.03.001 (accessed 15 May 2021).

18. Guo Y., Lin B., Wang W. Modeling of cutting forces with a serrated end mill. International Journal of Machine Tools and Manufacture, 2019, vol. 144, art. no. 103425. Available at: https://doi.org/10.1155/2019/1796926 (accessed 15 May 2021).

19. Dombovari Z., Altintas Y., Stepan G. The effect of serration on mechanics and stability of milling cutters. International Journal of Machine Tools and Manufacture, 2010, vol. 50, no. 6, pp. 511-520. Available at: https://doi.org/10.1016/j.ijmachtools.2010.03.006 (accessed 15 May 2021).

20. Sikhimbaev M.R., Sherov K.T., Zharkevich O.M., Sherov A.K., Tkachyova Y.O. Experimental studies of stabilization of boring cutter form - building top oscillation. Journal of Vibroengineering, 2012, vol. 14, no. 2, pp. 661-670.
21. Fedoseev S.N., Valuev D.V., Mamadaliev R.A., Sokolov P.N. Application of integrated methods to improve the technological properties of steel. Key Engineering Materials, 2017, vol. 736 . pp. 110-115. Available at: https://doi.org/10.4028/www.scientific. net/KEM.736.110 (accessed 15 May 2021).

22. Lukyanov A.V., Aleynikov D.P., Vishchenko M.V. Issledovanie prostranstvennykh vibratsiy i sil pri vysokoproizvoditelnom frezerovanii [Study of spatial vibrations and forces in high performance milling]. Transportation infrastructure of the Siberian region, 2017, vol. 2, pp. 598-603.

23. Bukreev V.G., Lyapushkin S.V., Edgulov M.M. Accuracy enhancement of automated system for multicomponent batching of bulk materials. MATEC Web of Conferences, 2015, vol. 37, 01012.

24. Bukreev V.G., Shandarova E.B., Rulevskiy V.M. Power supply system model of remote processing equipment. Bulletin of the Tomsk Polytechnic University. Geo Assets Engineering, 2018, vol. 329, no. 4, pp. 119-131. In Rus.

25. Rulevskiy V.M., Bukreev V.G., Kuleshova E.O. Shandarov S.M., Vasilyeva Yu.Z. The power supply system model of the process submersible device with AC power transmission over the cablerope. IOP Conference Series: Materials Science and Engineering, 2017, vol. 177 (1), 012098

26. Nizhegorodov A.I., Zvezdin A.V. Transformation of vermiculite energy into mechanical transformation energy during firing in electric furnaces with a «zero» module. Refractories and Industrial Ceramics, 2016, vol. 57, no. 3, pp. 239-245.

27. Vajda I., Glazyrin A., Ustinova I., Bolovin E. Influence of design methods a discrete model of separately excited DC motor on parameters estimation. Acta Polytechnica Hungarica, 2018, vol. 15, no. 6, pp. 219-233.

28. Korovin G.I., Zolotuhin D.V., Rozhkov P.S. Freza kontsevaya dlya obrabotki titanovykh splavov [End milling cutter for treatment of titanium alloys]. Patent RF no. 2014127926/02, 2016.

29. Korovin G.I., Petrushin S.I., Gubaidulina R.H. Machining of titanium alloys with wave milling cutters. Materials Science Forum, 2018, 927, pp. 79-85.

30. Frezy kontsevye iz bystrorezhushchey stali dlya obrabotki titanovykh splavov [HSS end mills for titanium alloys]. Available at: http:// pkmion.ru/catalog/dlya-obrabotki-titanovykh-splavov/710dlya-obrabotki-titanovykh-splavov/ (accessed 15 May 2021).

31. Gavrilin A.N., Vinogradov A.A., Serebryakov K.V. Vibroregistrator-F. Svidetelstvo o registratsii programmy dlya EVM Rossiyskoy Federatsii № 2014661189 [Federal registration certificate of the computer program of the Russian Federation No. 2014661189]. Registration date 24.10.2014

Received: 9 June 2021.

\section{Information about the authors}

Georgy I. Korovin, technical director, Industrial Company LLC « MION».

Alexey N. Gavrilin, Cand. Sc., associate professor, National Research Tomsk Polytechnic University.

Sergey I. Petrushin, Dr. Sc., professor, Kuzbass State Technical University.

Georgy I. Odnokopylov, Dr. Sc., professor, National Research Tomsk Polytechnic University.

Sergey N. Kladiev, Cand. Sc., associate professor, National Research Tomsk Polytechnic University. 